\title{
Elevated Plasma Adiponectin Level and Peripheral Blood Leukocyte Adiponectin Receptor Expression in Dogs Suffering from Insulin Deficiency
}

\author{
Nobuko Mori, Peter Lee, Ichiro Yamamoto and Toshiro Arai* \\ Department of Veterinary Science, School of Veterinary Medicine, Nippon Veterinary and Life Science University, \\ Tokyo, Japan
}

\begin{abstract}
The aim of this study is to determine and compare the effect of obesity or insulin deficiency on plasma adiponectin level and peripheral blood leukocyte (PBL) adiponectin receptor 1 and 2 (ADIPOR1 and -R2) expression in dogs. Obese and insulin deficient (ID) dogs demonstrated a significant reduction $(60 \%$ lower) and significant increase (2x higher) in circulating adiponectin level, respectively, as compared to normal controls. PBL ADIPOR1 and -R2 mRNA expression was also significantly higher in obese $(\mathrm{R} 1=333$ mean fold higher, $\mathrm{R} 2=64$ mean fold higher) and ID dogs preinsulin treatment $(\mathrm{R} 1=48$ mean fold higher, $\mathrm{R} 2=16$ mean fold higher) as compared to normal PBL. Insulin treatment reduced ADIPOR1 (3 mean fold higher) and -R2 (1.5 mean fold higher) expression back down closer to control PBL levels in ID animals. The upregulation of adiponectin receptor expression might reflect an increased need for adiponectin signaling; however, the increase may have a different implication between obese and ID dogs. This may be due to adiponectin's contradictory paradoxical dual role, having both anti-inflammatory and pro-inflammatory properties on peripheral leukocytes, especially on monocytes depending on the biological context and adiponectin isoform.
\end{abstract}

Keywords: Adiponectin, adiponectin receptor, anti-inflammation, dogs, pro-inflammation.

\section{INTRODUCTION}

Adiponectin is an adipokine involved in modulating whole-body metabolism and other vital functions related to inflammation and immune responses [1]. It is unique amongst adipokines, in part to its insulin-sensitizing action [2]. There is mounting evidence, based on human and rodent studies, suggesting that a reduced or low circulating level of adiponectin has been linked to several components of metabolic syndrome, including intra-abdominal body fat accumulation, and insulin resistance/Type 2 diabetes mellitus (T2DM) [3]. In addition, increased serum adiponectin has been associated with higher-all cause mortality in human patients with Type 1 DM (T1DM) and coexisting nephropathy [4]. Therefore, there appears to be a paradoxical inverse association between plasma adiponectin and complication risk in patients with T1DM compared with T2DM or obesity.

Currently, there is a paucity of studies focusing on adiponectin in dogs. Thus far, no studies have examined circulating adiponectin levels in insulin deficient dogs, and the relatively few studies focusing on adiponectin levels in naturally overweight and obese dogs have been inconclusive, purporting that adiposity is either negatively [5] or not associated [6-8] with adiponectin level. Obese dogs, like obese humans, have components of the metabolic syndrome such as insulin resistance [9]. However, it is widely

*Address correspondence to this author at the Department of Veterinary Science, School of Veterinary Medicine, Nippon Veterinary and Life Science University, Tokyo, Japan; Tel: +81422314151;

Fax:+81422317841; E-mail: tarai@nvlu.ac.jp recognized that atherosclerosis and T2DM are infrequently encountered and rare in dogs [10].

To date, ADIPOR1 and ADIPOR2 have been identified as receptors that mediate increased AMPK [11,12], peroxisome proliferator-activated receptor- $\alpha$ ligand activities, and fatty-acid oxidation and glucose uptake by adiponectin $[12,13]$. Both receptors are almost ubiquitously expressed in most tissues, albeit at different levels. ADIPOR1 is primarily expressed in testis, heart, and skeletal muscle, whereas ADIPOR2 is highly expressed in liver and testis and small intestine. Peripheral blood leukocytes (PBL) play a pivotal role in inflammation and atherosclerosis, and may serve as main sites whereby adiponectin exerts its antiinflammatory and anti-atherogenic effects $[11,14]$. Pang and Narendran [15] determined the distribution of adiponectin receptors on human peripheral mononuclear cells and found a similar distribution for ADIPOR1 and -R2, being approximately on $1 \%$ of T cells, $93 \%$ of monocytes, $47 \%$ of B cells, and $21 \%$ of NK cells. Since PBL are an easily accessible tissue source, they may serve as a valuable tissue source and to examine for alterations in ADIPOR1 and -R2 expression.

Therefore, the aim of this study was twofold. First, we sought to determine and compare adiponectin levels between control, naturally obese, and insulin deficient dogs. Second, since peripheral blood leukocytes (PBL), especially monocytes, are a major target of the actions of adiponectin, according to human and rodent studies [11], we sought to determine and compare mRNA expression levels of ADIPOR1 and -R2 on PBL of naturally obese and insulin deficient animals, since it has not yet been determined 
whether the expression of ADIPOR1 and -R2 are altered in physiological and pathological states in dogs.

\section{MATERIAL AND METHODS}

\section{Animals}

This study consisted of 18 dogs in total (Table 1). Six clinically healthy dogs, housed at the University, with a body condition score (BCS) of $<3$ on a 5 point scale (Beagles; F-5, $\mathrm{M}-1 ; 5-8 \mathrm{yrs}$ ) were grouped into the normal healthy control group of our study. Individual BCS of healthy dogs was determined and assessed by palpation and inspection on the five-point scale: (1) very thin, (2) underweight, (3) ideal, (4) overweight, and (5) obese. Six animals selected (3 Beagles, 3 Miniature Dachshund; $\mathrm{F}=4, \mathrm{M}=2$; 4-8 yrs) from 29 prospective dogs, which had visited the Tokyo Toyoda animal hospital from October 2009 to March 2010, became prospective obese study subjects. The identification of obese animals was mainly based on BCS and body weight. All obese animals were clinically diagnosed to be healthy and having a $\mathrm{BCS}>3$ by one veterinarian according to their anamnesis and based on clinical signs. The insulin deficient group comprised of 6 animals $(\mathrm{F}=1, \mathrm{M}=5 ; 1-10 \mathrm{yrs})$. The majority of dogs suffering from insulin deficiency had case histories of $\geq 5$ years, save for 1 young animal with a $<1$ year history. These animals were stabilized with long term insulin administration treatment. Lack of insulin treatment resulted in polydipsia, polyuria, glycosuria, high fasting plasma glucose, and extremely low fasting and non-fasting plasma insulin levels in these animals. Insulin deficient dogs were housed and treated at the NVLU animal clinic with 0.5 to $3.0 \mathrm{IU} / \mathrm{kg}$ body weight of either Detemir insulin or NPH insulin ( Novo Nordisk, Tokyo) injections, depending on each animal's individual diabetic state, twice daily ( 8 am and $8 \mathrm{pm}$ ).

\section{Blood Sampling}

Blood samples were collected from the cephalic vein of healthy and obese animals into heparinized plastic tubes at least $4 \mathrm{hrs}$ postprandial. Alternatively, with insulin deficient dogs, fasted pre-insulin treatment blood samples were obtained from the jugular vein and collected into heparinized plastic tubes prior to 8 am breakfast. Animals were given 15 minutes to finish their breakfast, before receiving their insulin treatments. Six hours post insulin injection, postinsulin treatment blood samples were collected again from the same dogs from the cephalic vein instead and collected into heparinized plastic tubes. Plasma of all subjects was recovered by centrifugation at $1200 \mathrm{~g}$ for $5 \mathrm{~min}$ at $4^{\circ} \mathrm{C}$ and subsequently stored at $-25^{\circ} \mathrm{C}$ until further use.

\section{Plasma Enzyme and Hormone and Cytokine Analysis}

Alkaline phosphatase (ALP), alanine aminotransferase (ALT), aspartate aminotransferase (AST), lactate dehydrogenase (LDH) activities, blood urea nitrogen (BUN), creatinin (CRE), glucose (GLU), total cholesterol (T-Cho), total protein(TP) and triglyceride(TG) concentrations were measured using an Olympus AU680 auto analyzer (Olympus Corporation, Tokyo) with the manufacturer's reagents. Adiponectin, free fatty acid (FFA), insulin, were determined with the following commercial kits respectively: Dog

Table 1. Profiles, Signalment and Treatment Protocol of 18 Dogs

\begin{tabular}{|c|c|c|c|c|c|c|}
\hline & Breed & Gender & Age (Years) & Insulin Treatment Quantity (IU/kg) & Cause of Insulin Deficiency & History of T1DM \\
\hline \multirow{6}{*}{$\stackrel{80}{\stackrel{0}{0}}$} & Miniature Dachshund & Male & 1 & NPH insulin 2 & Natural, spontaneous & $<1 \mathrm{yr}$ \\
\hline & Miniature Dachshund & Male & 6 & Detemir insulin 1 & Natural, spontaneous & $>5 \mathrm{yrs}$ \\
\hline & Miniature Schnauzer & Female & 5 & Detemir insulin 0.5 & Natural, spontaneous & $>5 y r s$ \\
\hline & Beagle & Male & 8 & Detemir insulin 3 & Chemical, STZ treatment & $>5 \mathrm{yrs}$ \\
\hline & Beagle & Male & 8 & Detemir insulin 2.5 & Chemical, STZ treatment & $>5 \mathrm{yrs}$ \\
\hline & Beagle & Male & 10 & Detemir insulin 1.5 & Chemical, STZ treatment & $>5 \mathrm{yrs}$ \\
\hline \multirow{6}{*}{ 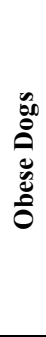 } & Miniature Dachshund & Male & 7 & \multirow{6}{*}{ N/A } & \multirow{6}{*}{ N/A } & \multirow{6}{*}{ N/A } \\
\hline & Miniature Dachshund & Male & 4 & & & \\
\hline & Miniature Dachshund & Female & 8 & & & \\
\hline & Beagle & Female & 7 & & & \\
\hline & Beagle & Female & 7 & & & \\
\hline & Beagle & Female & 8 & & & \\
\hline \multirow{6}{*}{ 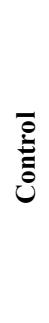 } & Beagle & Female & 5 & \multirow{6}{*}{ N/A } & \multirow{6}{*}{ N/A } & \multirow{6}{*}{ N/A } \\
\hline & Beagle & Female & 5 & & & \\
\hline & Beagle & Female & 6 & & & \\
\hline & Beagle & Female & 7 & & & \\
\hline & Beagle & Female & 7 & & & \\
\hline & Beagle & Male & 8 & & & \\
\hline
\end{tabular}


Adiponectin ELISA Kit (CircuLex Co., Ltd. Nagano), NEFA-C test Wako (Wako Pure Chemical Industries, Inc., Tokyo), Lbis Dog Insulin kit (SHIBAYAGI Co., Gunma).

\section{Quantitative Real-Time PCR Analysis of mRNA}

Total leukocyte RNA from dog samples was extracted and isolated using a PAX gene Blood RNA V.2 kit (PreAnalytiX $\mathrm{GmbH}$ ) and ISOGEN (Nippon Gene) according to the manufacturer's instructions. Total RNA was reverse-transcribed to cDNA using a QuantiTect Reverse Transcription Kit (QIAGEN, Tokyo, Japan), according to the manufacturer's instructions. Expression levels of ADIPOR1 and R2 mRNA were determined by quantitative RT-PCR (qRT-PCR), in triplicate, using self designed primers as described in Table $\mathbf{2}$. Dog-specific $\beta$-actin was selected as a housekeeping gene for our experiments. qRT-PCR reactions were performed using SYBER Premix EX Taq II (Takara, Tokyo, Japan) and an ABI 7300 Real Time PCR system Sequence Detection System (Applied Biosystems, Foster City, CA).

Absolute quantification was performed as described by Whelan et al., [16]. In brief, linear amplification curves (Standard curves) for each gene (ADIPOR1,-R2, and $\beta$ actin) were generated from tenfold serial dilutions of cloned and sequenced plasmid DNAs. mRNA expression was calculated and expressed as copy number/ng of cDNA input.

Relative gene expression values were calculated by the relative standard curve method for quantification as described in the ABI 7300 Real Time PCR system Sequence Detection System accompanying chemistry guide. All target genes were normalized to $\beta$-actin in order to obtain normalized expression results, and subsequently compared to normal to determine relative $n$-fold difference to normal.

\section{Statistical Analysis}

Plasma analytic data values are expressed as mean with $\min / \mathrm{max}$ range values. mRNA expression data values are represented as mean \pm STD (standard deviation). In order to assess significance between qRT-PCR groups, the MannWhitney U-Test was employed for data with non-normal distribution. Holm-Sidak One Way ANOVA was utilized to perform multiple group comparison of plasma analytic values. Statistical significance was set at $\mathrm{P}<0.05$ for both the U-Test and One Way ANOVA. All tests were conducted using Sigmaplot analysis software (Sigmaplot 11.0, Build 11.0.077; Systat Software Inc., San Jose, CA).

\section{RESULTS}

\section{Plasma Enzyme and Hormone and Cytokine Analysis}

Results for all dog groups are presented in Table $\mathbf{3}$. The obese group demonstrated some significant (Holm-Sidak One Way ANOVA, $\mathrm{p}<0.05$ ) differences as compared to the control group. FFA, insulin and LDH were 2.0 fold, 3.3 fold, and 4.1 fold higher respectively, whereas adiponectin level was significantly lower $(75 \%$ less $)$ in the obese group as compared to the control group. The insulin deficient (ID) group also demonstrated some significant differences, mainly constituting an increase in adiponectin (2 fold), ALP (2.3 fold), ALT (4.2 fold), AST (1.7 fold), insulin (11 fold), and a decrease in FFA (50\% reduction), glucose $(40 \%$ reduction), and $\mathrm{LDH}$ (17\% reduction) as compared to control animals. Interestingly, pre insulin treatment values for adiponectin and FFA were significantly higher being $\sim 18 \%$ and $\sim 160 \%$ greater whereas other values did not significantly differ (data not shown).

\section{Quantitative ADIPOR1 and ADIPOR2 mRNA Expression on Peripheral Blood Leukocytes}

ADIPOR1 and -R2 mRNA expression levels on peripheral blood leukocytes (PBL) were assessed quantitatively by copy number/ng cDNA using qRT-PCR for all groups (Fig. 1). As compared to control PBL, a similar pattern was observed between ADIPOR1 and -R2 mRNA expression in both obese and ID PBL in which ADIPOR1 expression was always greater than -R2. However, normal animal PBL demonstrated an ADIPOR1/R2 ratio of $\sim$, while the ratio was almost double for obese PBL $(\sim 13)$, whereas the ratio was very similar to control in ID PBL $(\sim 6.5)$. However, the ratio $(\sim 22)$ was $\sim 3.5 x$ higher in ID PBL pre-insulin (data not shown).

\section{Relative ADIPOR1 and ADIPOR2 mRNA Expression Level as Compared to Normal PBL}

mRNA expression levels of ADIPOR1 and -R2 on PBL of obese and ID animals were compared against normal PBL (Fig. 2). Obese dog peripheral leukocytes demonstrated significantly higher (Mann Whitney U-test, $\mathrm{p}<0.05$ ) mRNA levels of both adiponectin receptors (ADIPOR $1=333$ mean fold, ADIPOR2=64 mean fold) as compared to control animal PBL. Pre-insulin ID PBL, also demonstrated significantly higher (Mann Whitney U-test, $\mathrm{p}<0.05)$ mRNA levels for ADIPOR1 (48 mean fold higher) and -R2 (16 mean fold) as compared to control PBL. Insulin treatment appeared to reduce both ADIPOR1 (3 mean fold higher) and -R2 (1.5 mean fold higher) mRNA expression levels by $90-95 \%$, bringing them both back

Table 2. Primer Sequences Used in this Study

\begin{tabular}{|c|c|c|c|c|}
\hline \multicolumn{2}{|l|}{ Primer Sequences for Real-time PCR } \\
\hline Probe & Suquencing Length of PCR Products (bp) & Primer Type & GeneBank Acc. No. & Primer Sequences (5'-3') \\
\hline \hline Adiponectin receptor 1 & 247 & $\begin{array}{l}\text { Forward } \\
\text { Reverse }\end{array}$ & XM_843263.1 & $\begin{array}{c}\text { cttctactgctccccacagc } \\
\text { catcacagccatgaggaaga }\end{array}$ \\
\hline Adiponectin receptor 2 & 146 & $\begin{array}{l}\text { Forward } \\
\text { Reverse }\end{array}$ & XM_534929.2 & $\begin{array}{c}\text { tccacaaccttgcttcatct } \\
\text { tgattccactcagaccaagg }\end{array}$ \\
\hline$\beta$-actin & 129 & Forward & AF021873.2 & gccaaccgtgagaagatgact \\
& & & & Reverse \\
& & & &
\end{tabular}


Table 3. Clinical Characteristics and Plasma Metabolite Concentrations

\begin{tabular}{|c|c|c|c|c|}
\hline & Clinical Parameters & $\begin{array}{c}\text { Control } \\
(\mathrm{n}=6)\end{array}$ & $\begin{array}{l}\text { Obese } \\
(n=6)\end{array}$ & $\begin{array}{c}\text { Insulin Deficient } \\
(n=6)\end{array}$ \\
\hline & Age (years) & $\begin{array}{c}7 \\
(1-10)\end{array}$ & $\begin{array}{c}7 \\
(4-8)\end{array}$ & $6.5(5-8)$ \\
\hline \multirow{2}{*}{ 充 } & Glucose (mg/dl) & $\begin{array}{c}97.5 \\
(81.2-105.0)\end{array}$ & $\begin{array}{c}89 \\
(70.1-99.0)\end{array}$ & $\begin{array}{c}58 \\
(49.0-172.0)\end{array}$ \\
\hline & Insulin (ng/ml) & $\begin{array}{c}0.72 \\
(0.47-1.68)\end{array}$ & $\begin{array}{c}2.39 * \\
(0.88-4.26)\end{array}$ & $\begin{array}{c}9.03 * \\
(0.57-17.46)\end{array}$ \\
\hline \multirow{4}{*}{$\begin{array}{l}\frac{\vec{n}}{\overline{0}} \\
\frac{0}{0}\end{array}$} & Adiponectin (mg/ml) & $\begin{array}{c}29.00 \\
(8.63-37.60)\end{array}$ & $\begin{array}{c}6.52 * \\
(4.36-18.33)\end{array}$ & $\begin{array}{c}48.18^{* *} \\
(15.70-69.37)\end{array}$ \\
\hline & Free fatty acid (mEq/l) & $\begin{array}{c}0.44 \\
(0.29-0.73)\end{array}$ & $\begin{array}{c}0.90^{*} \\
(0.40-2.91)\end{array}$ & $\begin{array}{c}0.23 * * \\
(0.18-0.29)\end{array}$ \\
\hline & Total Cholesterol (mg/dl) & $\begin{array}{c}195.47 \\
(188.49-221.30)\end{array}$ & $\begin{array}{c}168.06 \\
(143.56-303.59)\end{array}$ & $\begin{array}{c}170.00 \\
(136.0-206.0)\end{array}$ \\
\hline & Triglycerides (mg/dl) & $\begin{array}{c}72.38 \\
(49.42-122.47)\end{array}$ & $\begin{array}{c}73.91 \\
(27.87-123.98)\end{array}$ & $\begin{array}{c}49.50 \\
(37.0-72.0)\end{array}$ \\
\hline \multirow{7}{*}{$\stackrel{\bar{d}}{:}$} & Alanine aminotransferase (U/l) & $\begin{array}{c}34 \\
(20.0-47.0) \\
\end{array}$ & $\begin{array}{c}29 \\
(22.0-33.0) \\
\end{array}$ & $\begin{array}{c}144 *, * * \\
(74.0-199.0) \\
\end{array}$ \\
\hline & Alkaline Phosphatase (U/1) & $\begin{array}{c}187 \\
(143.0-217.0)\end{array}$ & $\begin{array}{c}295 \\
(67.0-486.0)\end{array}$ & $\begin{array}{c}439 * * * \\
(404.0-780.0)\end{array}$ \\
\hline & Aspartate aminotransferase (U/l) & $\begin{array}{c}24.8 \\
(22.0-28.3)\end{array}$ & $\begin{array}{c}27.5 \\
(18.1-50.6)\end{array}$ & $\begin{array}{c}41.0^{* *} \\
(28.5-56.8)\end{array}$ \\
\hline & Blood Urea Nitrogen (mg/dl) & $\begin{array}{c}14.6 \\
(13.8-24.6)\end{array}$ & $\begin{array}{c}18.7 \\
(12.2-40.8)\end{array}$ & $\begin{array}{c}15.7 \\
(13.0-20.0)\end{array}$ \\
\hline & Creatinine (mg/dl) & $\begin{array}{c}0.59 \\
(0.50-0.97) \\
\end{array}$ & $\begin{array}{c}0.64 \\
(0.25-2.09) \\
\end{array}$ & $\begin{array}{c}1.05 \\
(1.01-1.42) \\
\end{array}$ \\
\hline & Lactate dehydrogenase (U/l) & $\begin{array}{c}136.5 \\
(94.0-228.0)\end{array}$ & $\begin{array}{c}562 * \\
(51.0-810.0)\end{array}$ & $\begin{array}{c}100^{* *} \\
(64.0-173.0)\end{array}$ \\
\hline & Total Protein $(\mathrm{g} / \mathrm{dl})$ & $\begin{array}{c}6.4 \\
(5.7-6.9)\end{array}$ & $\begin{array}{c}6.5 \\
(5.7-10.0)\end{array}$ & $\begin{array}{c}5.3^{* *} \\
(4.9-5.6)\end{array}$ \\
\hline
\end{tabular}

Values are presented as median with (range).

*Denotes significance when compared against control group $(\mathrm{p}<0.05$, Holm-Sidak one way ANOVA).

$* *$ Denotes significance when compared against obese group $(\mathrm{p}<0.05$, Holm-Sidak one way ANOVA)

down closer to control PBL levels.

\section{DISCUSSION}

Adiponectin research in dogs is not as extensive as that in humans and mice. Currently, one of the main motivating forces behind adiponectin research in dogs is the potential role of adipokines as biomarkers of obesity-associated disease in companion animals. In three of the most recently published canine studies, $29-34 \%$ of dogs were classed as overweight and $5-8 \%$ were judged to be obese [17]. As compared to healthy control animals, an $80 \%$ reduction in total plasma adiponectin level was observed in overweight dogs, in our study. This trend is in agreement with previously published adiponectin based studies in humans and rodents [3,18]. Moreover, our finding is supported by previously published adiponectin studies in dogs $[5,19]$, but contradicts other canine studies purporting that adiposity is not associated with adiponectin level [6-8].

Two possible factors which may confound the relationship between adiposity and adiponectin concentration in dogs have been advanced. First, neutering status in obese dogs may be a factor [20], but its mechanism is unknown. Accordingly, the studies that have found an association between adiponectin concentration and adiposity, sampled 
predominantly intact dogs $[5,19]$, whereas the studies that found no effect involved predominantly neutered dogs
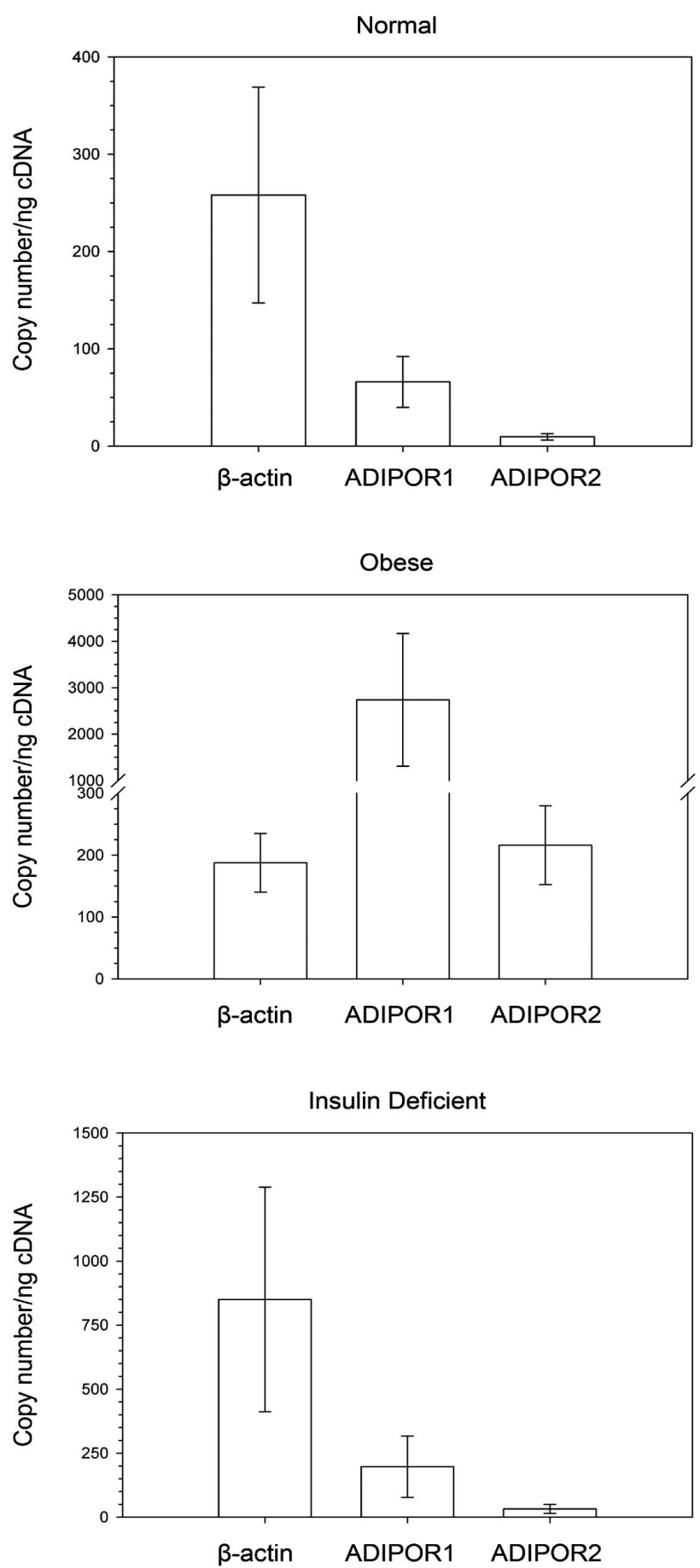

Fig. (1). Quantitative adiponectin receptor 1 (ADIPOR1) and adiponectin receptor 2 (ADIPOR2) mRNA expression levels determined by RT-PCR in peripheral blood leukocytes (PBL) from control (top panel, $n=6$ ), obese (middle panel, $n=6$ ), and insulin deficient (ID) (bottom panel, $\mathrm{n}=6$ ) dogs. Standard curves for each gene (ADIPOR1,-R2, and $\beta$-actin) were generated from tenfold serial dilutions of cloned and sequenced plasmid DNAs. mRNA expression was calculated and expressed as copy number/ng of cDNA input. Values are expressed as mean \pm STD.

$[6,8]$ or were in a country where neutering is common [7]. Unfortunately, the neutering status of the client owned animals used in our study were not detailed and not provided to us. Second, the type of obesity, natural occurring obesity,

A.

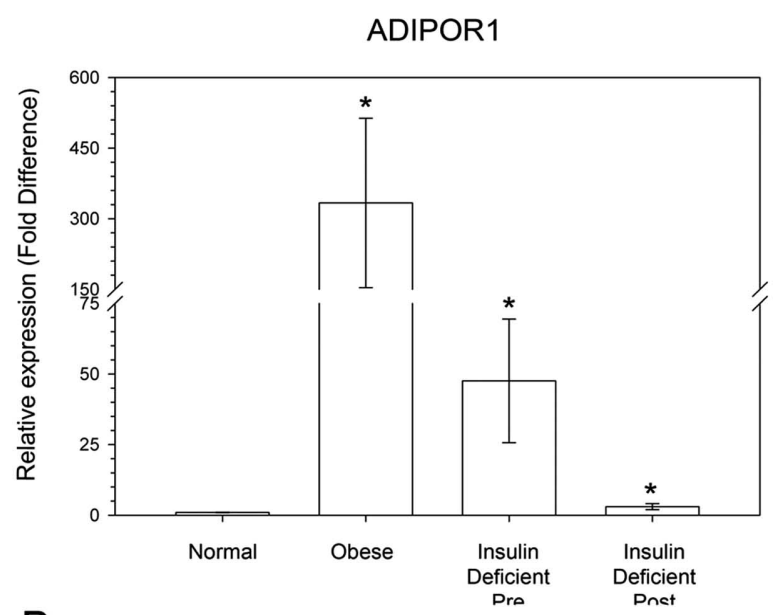

B.

\section{ADIPOR2}

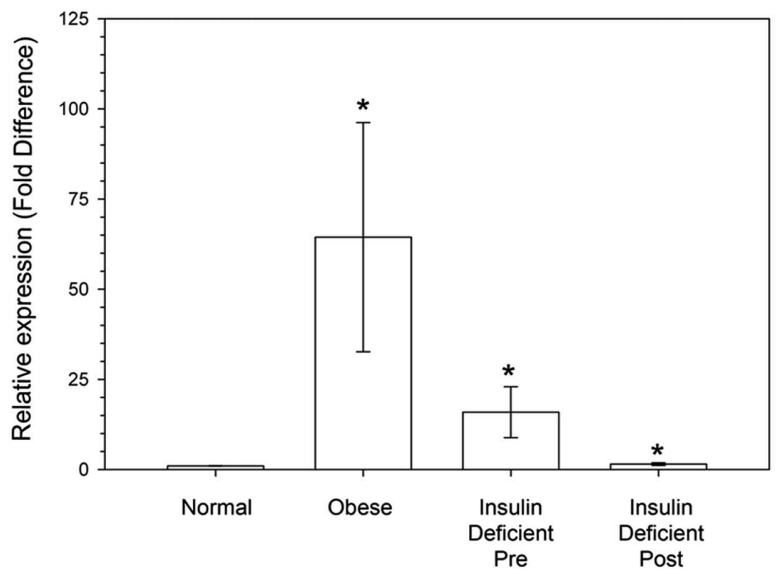

Fig. (2). Relative adiponectin receptor 1 (ADIPOR1, panel A) and adiponectin receptor 2 (ADIPOR2, panel B) mRNA expression levels on peripheral blood leukocytes (PBL) of obese $(n=6)$ and insulin deficient (ID) $(n=6)$ animals as compared to controls $(n=6)$. In the case of ID dogs, we obtained pre-and post-insulin treatment blood to determine the effect of insulin. Results are expressed as relative mRNA levels in arbitrary unit \pm SD as compared to control level, with values being the mean of three independent experiments and control level set at 1 . Single asterisk denotes higher significance $(\mathrm{p}<0.05$, Mann-Whitney U-Test $)$ when compared against control dogs.

as opposed to obesity acutely induced with a high-fat diet, may also be important [21]. Previous studies which did not observe any reduction in total serum adiponectin level employed naturally obese client owned animals $[6,7,8]$. However, our study and that of Tvarijonaviciute et al., [19] also employed naturally obese client owned animals and observed a decrease in total adiponectin, thus countering the notion of obesity type effect.

One other alternative factor which needs to be considered in light of indifference in obesity type is diet. Since obese condition has already been diagnosed in obese animals, the majority of these animals have probably been ordered to consume particular diets to try and ameliorate their 
condition. Dietary management has been considered an alternative means of modulating adiponectin levels and can be an effective therapeutic means of increasing adiponectin levels in humans [22]. For example, based on human studies, daily intake of fish or omega-3 supplementation can increase adiponectin levels by $14-60 \%$, fiber supplementation can lead to a $60-115 \%$ increase in adiponectin levels, and weight loss achieved with a low-calorie diet plus exercise can increase adiponectin levels in the range of $18-48 \%$ [22]. In contrast, carbohydrate-rich and high-fat diets appear to decrease plasma adiponectin level [23]. Hence, details concerning the three aforementioned factors (neuter status, type of obesity, and diet) may be required on any future obesity studies involving adiposity and adiponectin in dogs, in order to better explain their results, since the relationship between adiposity and adiponectin in dogs has yet to be unequivocally demonstrated.

Unlike cats or humans, dogs have not been documented to develop T2DM [10], therefore the majority of diabetes cases in appear to be primarily attributed to insulin deficiency, which can occur from immune mediated $\beta$-cell loss and/or exocrine pancreatic disease, or insulin antagonism (eg. dioestrus diabetes and other endocrinopathy) thereby making it more similar to T1DM in nature. With respect to insulin deficiency (ID), an overall 2 fold increase in plasma adiponectin level in ID dogs, was observed as compared to control animals in our study. This trend is in agreement with previously published human studies correlating higher adiponectin concentration in patients with T1DM [24, 25]. It is currently unknown as to why T1DM human patients have significantly higher circulating adiponectin levels as compared to healthy individuals. In fact, lower fasting triglyceride levels have been observed, while post-prandial triglyceride accumulation was prevented after consuming a high fat diet in human T1DM patients with high adiponectin levels [26]. Coincidentally, the ID dogs in our study demonstrated $\sim 50 \%$ lower post-prandial circulating TG levels as compared to controls.

It has not yet been determined whether the expression of ADIPOR1 and -R2 are altered in physiological and pathological states in dogs. Therefore, adiponectin receptor expression on PBL was examined in obese and ID animals. Between both adiponectin receptors, ADIPOR1 expression is more dominant as evidenced in all of our groups regardless of physiological and pathological states. It has been hypothesized that in the steady state, adiponectin level is lower, but its receptors level is higher in obese people than in control subjects [27]. Adiponectin receptor (ADIPOR1 and ADIPOR2) mRNA expression level from obese dog PBL was generally upregulated as compared to control animals. This is in agreement with a human based study examining mRNA adiponectin receptor expression on peripheral blood mononuclear cells, in which PMBC from obese humans also had higher expression levels of ADIPOR1 and -R2, respectively as compared to control PBMC [28].

It has also been purported that the expression of $\mathrm{ADIPOR} 1 / \mathrm{R} 2$ in vivo may be inversely correlated with plasma insulin levels [29], and that insulin negatively regulates the expression levels of adiponectin receptors and insulin sensitivity in muscle and adipose according to mice studies [26]. The inhibitory effect of insulin especially on ADIPOR1 expression has been proposed to be mediated by phosphoinositide 3-kinase (PI3-kinase) and Foxo1 pathways [29, 30] targeting a strong repressor element, termed nuclear inhibitory protein [31], within the putative insulin-responsive region in the AdipoR1 promoter. As such, we compared ADIPOR1/R2 expression on PBL from ID animals pre and post insulin treatment to determine whether this held true for dogs also. Interestingly, a similar trend of receptor expression upregulation in obese animal PBL was also observed with PBL from insulin deficient animals (ID) pre insulin treatment. The mRNA expression level of ADIPOR1 and ADIPOR2 was upregulated $\sim 48$ and $\sim 16$ mean fold higher, respectively as compared to control animals. After insulin treatment, the mRNA expression level of ADIPOR1 and ADIPOR2 was significantly downregulated to $\sim 3$ and $\sim 1.5$ mean fold higher, respectively as compared to control animals, thus bringing expression levels close to that observed in control animal PBL.

This reduction confirms the negative effect of insulin on ADIPOR1/R2 expression in dogs and is supported by results from a study demonstrating that insulin treatment effectively brought back plasma adiponectin level and ADIPOR1 mRNA expression levels in muscle back to control levels in rats suffering from Streptozotocin-induced Type 2 diabetes [32]. Coincidentally, plasma adiponectin level was reduced by $\sim 16 \%$ after insulin treatment (data not shown). Intensifying glycemic control with insulin has been shown to reduce total adiponectin moderately in T2, but not T1DM human patients [26]. Moreover, this reduction in adiponectin receptor expression may be related to alteration in systemic inflammation status, due to insulin, which may be reflected by plasma FFA levels. FFAs are known potent physiological inducers of TNF- $\alpha$ production in adipocytes [33] and a pro-inflammatory response in $\beta$-cell islets [34]. Obese animals demonstrated 2x more plasma FFA as compared to control animals; whereas ID animals demonstrated $50 \%$ higher plasma FFA pre-insulin treatment, and 50\% less plasma FFA post-insulin treatment, as compared to control animals. Insulin was able to reduce the amount of FFA by $80 \%$ of pre-insulin values.

Our study has a number of limitations however. The study employed a small number of animals in each group $(n=6)$ since this was a preliminary study. As such, caution is required when assessing the influence of obesity or insulin deficiency on ADIPOR1,-R2 expression and interpreting our results. Only total adiponectin was measured using a commercial kit, therefore adiponectin isoforms were not subtyped. Adiponectin has four independent variant forms at the molecular level: LMW, MMW, HMW, and globular. It has been reported that ADIPOR1 has greater affinity for globular and LMW adiponectin, while ADIPOR2 exhibits greater affinity for MMW and HMW forms of adiponectin [35]. Moreover, it is known that HMW adiponectin has pro-inflammatory effects leading to increased IL- 8 production and IL-6 secretion by human macrophages and monocytes, respectively, whereas only LMW adiponectin has anti-inflammatory effects since it can down-regulate IL- 6 production and induce IL-10 synthesis by monocytes [36-38]. However, since plasma adiponectin was not subtyped, it is unknown which isoform may be contributing to the overall increase or reduction in adiponectin in ID or obese dogs, respectively, and subsequently what implication this outcome would have in relation to the ADIPOR1,-R2 profile expression exhibited by PBL. We plan to subtype adiponectin in all future adiponectin studies. 


\section{ACKNOWLEDGEMENTS}

This work was supported in part by the Strategic Research Base Development Program for Private Universities from the Ministry of Education, Culture, Sports, Science and Technology of Japan (MEXT), 2008-2012.

\section{CONFLICT OF INTEREST STATEMENT}

None of the authors has any financial or personal relationships that could inappropriately influence or bias the content of the paper.

\section{REFERENCES}

[1] Berg AH, Scherer PE. Adipose tissue, inflammation, and cardiovascular disease. Circ Res 2005; 96(9): 939-49

[2] Berg AH, Combs TP, Du X, Brownlee M, Scherer PE. The adipocyte secreted protein Acrp30 enhances hepatic insulin action. Nat Med 2001; 7(8): 947-53.

[3] Chandran M, Ciaraldi T, Phillips SA, Henry RR. Adiponectin: more than just another fat cell hormone? Diabetes Care 2003; 26(8): 2442-50

[4] Jorsal A, Tarnow L, Frystyk J, et al. Serum adiponectin predicts allcause mortality and end stage renal disease in patients with type I diabetes and diabetic nephropathy. Kidney Int 2008; 74(5): 649-54.

[5] Ishioka K, Omachi A, Sagawa M, et al. Canine adiponectin: cDNA structure, mRNA expression in adipose tissues and reduced plasma levels in obesity. Res Vet Sci 2006; 80(2): 127-32.

[6] Verkest KR, Fleeman LM, Morton JM, Ishioka K, Rand JS. Compensation for obesity-induced insulin resistance in dogs: assessment of the effects of leptin, adiponectin, and glucagon-like peptide-1 using path analysis. Domest Anim Endocrinol 2011; 41(1): 24-34.

[7] Wakshlag J, Struble A, Levine C, et al. Effects of weight loss on adipokines and markers of inflammation in dogs [abstract]: The Waltham International Nutritional Sciences Symposium Pet Nutrition Art or Science? University of Cambridge, Cambridge, United Kingdom. 2010

[8] German AJ, Hervera M, Hunter L, et al. Improvement in insulin resistance and reduction in plasma inflammatory adipokines after weight loss in obese dogs. Domest Anim Endocrinol 2009; 37(4): 214 26.

[9] Bergman RN, Kim SP, Catalano KJ, et al. Why visceral fat is bad: mechanisms of the metabolic syndrome. Obesity (Silver Spring) 2006; 14 (Suppl 1): 16S-19S.

[10] Ettinger SJ, Feldman EC. Textbook of Veterinary Internal Medicine. $5^{\text {th }}$ ed. WB Saunders, Philadelphia, 2000; pp. 70-2.

[11] Yamauchi T, Kamon J, Minokoshi Y, et al. Adiponectin stimulates glucose utilization and fatty-acid oxidation by activating AMP activatedprotein kinase. Nat Med 2002; 8(11): 1288-95.

[12] Tomas E, Tsao TS, Saha AK, et al. Enhanced muscle fat oxidation and glucose transport by ACRP30 globular domain: acetyl-CoA carboxylase inhibition and AMP-activated protein kinase activation. Proc Natl Acad Sci USA 2002; 99(25): 16309-13

[13] Yamauchi T, Kamon J, Ito Y, et al. Cloning of adiponectin receptors that mediate antidiabetic metabolic effects. Nature 2003; 423(6941): 762-9.

[14] Ouchi N, Kihara S, Arita Y, et al. Novel modulator for endothelial adhesion molecules: adipocyte derived plasma protein adiponectin. Circulation 1999; 100(25): 2473-6.

[15] Pang TT, Narendran P. The distribution of adiponectin receptors on human peripheral blood mononuclear cells. Ann NY Acad Sci 2008; 1150: 143-5.

[16] Whelan JA, Russell NB, Whelan MA. A method for the absolute quantification of cDNA using real-time PCR. J Immunol Methods 2003; 278(1-2): 261-9.
[17] Lund EM, Armstrong PJ, Kirk CA, Klausner JS. Prevalence and risk factors for obesity in adult dogs from private US veterinary practices. Intern J Appl Res Vet Med 2006; 4(2): 177-86.

[18] Weigert J, Neumeier M, Wanninger J, et al. Reduced response to adiponectin and lower abundance of adiponectin receptor proteins in type 2 diabetic monocytes. FEBS Lett 2008; 582(12): 1777-82.

[19] Tvarijonaviciute A, Martínez-Subiela S, Ceron JJ. Validation of 2 commercially available enzyme-linked immunosorbent assays for adiponectin determination in canine serum samples. Can J Vet Res 2010; 74: 279-85.

[20] Verkest KR, Rose FJ, Fleeman LM, et al. Adiposity and adiponectin in dogs: investigation of causes of discrepant results between two studies. Domest Anim Endocrinol 2011; 41(1): 35-41.

[21] Verkest KR, Rand JS, Fleeman LM, et al. Distinct adiponectin profiles might contribute to differences in susceptibility to type 2 diabetes in dogs and humans. Domest Anim Endocrinol 2011; 41(2): 67-73.

[22] Silva FM, de Almeida JC, Feoli AM. Effect of diet on adiponectin levels in blood. Nutr Rev 2011; 69(10): 599-612.

[23] Pischon T, Girman CJ, Rifai N. Association between dietary factors and plasma adiponectin concentration in men. Am J Clin Nutr; 2005; 81(4): 780-6.

[24] Imagawa A, Funahashi T, Nakamura T, et al. Elevated serum concentration of adipose-derived factor, adiponectin, in patients with type 1 diabetes. Diabetes Care 2002; 25(9): 1665-6.

[25] Maruyama C, Ishibashi R, Araki R. Koike S, Hirose H, Maruyama T. HMW-adiponectin associates with triglyceride concentrations in type 1 diabetic patients. J Atheroscler Thromb 2009; 16(3): 207-16.

[26] Tsuchida A, Yamauchi T, Ito Y, et al. Insulin/Foxo1 pathway regulates expression levels of adiponectin receptors and adiponectin sensitivity. J Biol Chem 2004; 279(29): 30817-22.

[27] Wolfe BE, Jimerson DC, Orlova C, Mantzoros CS. Effect of dieting on plasma leptin, soluble leptin receptor, adiponectin and resistin levels in healthy volunteers. Clin Endocrinol (Oxf). 2004; 61(3): 332-8.

[28] Neels JG, Olefsky JM. Inflamed fat: what starts the fire? J Clin Invest 2006; 116(1): 33-5.

[29] Leth H, Andersen KK, Frystyk J, et al. Elevated levels of highmolecular-weight adiponectin in type 1 diabetes. J Clin Endocrinol Metab 2008; 93(8): 3186-91.

[30] Fang X, Palanivel R, Zhou X, et al. Hyperglycemia- and hyperinsulinemia-induced alteration of adiponectin receptor expression and adiponectin effects in L6 myoblasts. J Mol Endocrinol 2005; 35(3): 465-76.

[31] Sun X, He J, Mao C, et al. Negative regulation of adiponectin receptor 1 promoter by insulin via a repressive nuclear inhibitory protein element. FEBS Lett 2008; 582(23-24): 3401-7.

[32] Böni-Schnetzler M, Boller S, Debray S, et al. Free fatty acids induce a proinflammatory response in islets via the abundantly expressed interleukin-1 receptor I. Endocrinology 2009; 150(12): 5218-29.

[33] Kadowaki T, Yamauchi T. Adiponectin and adiponectin receptors. Endocr Rev 2005; 26(3): 439-51.

[34] Al-Hasehm F, Ibrahim I, Bastawy N, et al. Effect of insulin on adiponectin and adiponectin receptor-1 expression in rats with streptozotocin-induced Type 2 diabetes. J Health Sci 2011; 57(4): 33440.

[35] Lovren F, Pan Y, Quan A, et al. Adiponectin primes human monocytes into alternative anti-inflammatory M2 macrophages. Am J Physiol Heart Circ Physiol 2010; 299(3): H656-63.

[36] Neumeier M, Weigert J, Schäffler A, et al. Different effects of adiponectin isoforms in human monocytic cells. J Leukoc Biol 2006; 79(4): 803-8.

[37] Ohashi K, Parker JL, Ouch N, et al. Adiponectin promotes macrophage polarization toward an anti-inflammatory phenotype. J Biol Chem 2010; 285(9): 6153-60.

[38] Shapiro L, Scherer PE. The crystal structure of a complement-1q family protein suggests an evolutionary link to tumor necrosis factor. Curr Biol $1998 ; 8(6): 335-8$ 